\section{The importance of set-pieces in soccer: Russia 2018 FIFA World Cup analysis}

\section{Futbolda duran toplarin önemi: Rusya 2018 FIFA Dünya Kupasının analizi}

\author{
Kemal Göral $^{1}$
}

\begin{abstract}
Penalties, free-kicks (direct-indirect), throw-ins, corner kicks and goal kicks are known to be as set-pieces. In this study; examination of setpieces' effects on scoring goals were targeted and, in this context, the competitions of Russia 2018 FIFA World Cup were analyzed. As a part of this study, 64 competitions of Russia 2018 FIFA World Cup were analyzed depending on penalties and set-pieces (free-kicks, corner kicks and throw-ins etc.) ending with goals. "EAnalyze Soccer" computer program were used to analyze the set-piece organizations in the competitions at the tournament. The findings were registered and interpreted via SPSS (18.0) program upon calculations of frequencies and percentages. $21(\% 12.43)$ of the all goals scored in this tournament were from penalties, 23 (\%13.61) from free-kicks, 22 (\%13.02) from corner kicks and $2(\% 1.18)$ from throw-ins. Throughout the Russia 2018 FIFA World Cup, 169 goals were scored in total with an average of 2.64 goals per game and, $68(\% 40.24)$ of all the goals were recorded as score following the setpieces. In the tournament, $21(\% 72.41)$ penalties out of 29 resulted with goals. A major part of the goals scored from corner kicks were completed via headings $(\% 77.27)$ and rest with kicks (\%22.73). A great amount of completing goal scoring kicks following corner kicks were recorded with right foot $(\% 81.82)$ and the rest with left foot (\%18.18). As a result, the goals
\end{abstract}

\section{Özet}

Penaltılar, serbest vuruşlar (direkt-endirekt), taç atışları, köşe vuruşları ve kale vuruşları, futbolda duran toplar olarak adlandirılmaktadır. Bu çalışma, futbolda duran topların, maçlarda atılan goller üzerindeki etkisinin incelenmesi ve bu kapsamda Rusya'da düzenlenen 2018 FIFA Dünya kupasındaki müsabakaların analiz edilmesi amacıyla yapılmıştır. Çalışmada, 2018 FIFA Futbol Dünya kupasinda oynanan 64 müsabakada, kullanilan penaltılar ile duran top sonras1 golle sonuçlanan serbest vuruş, köşe vuruşu ve taç atışlarının analizi yapılmıştur. Turnuvada oynanan müsabakalarda, kullanilan duran top organizasyonlarinı (penaltı, serbest vuruş, köşe vuruşları ve taç atısları) analiz etmek için bilgisayar ortaminda "e-analyze soccer" futbol analiz program kullanılmıştır. Elde edilen veriler SPSS 18.0 programında kaydedilmiş, frekans ve yüzde değerleri hesaplandıktan sonra yorumlanmıştır. Turnuvada atulan gollerin $\% 12.43$ ü (21 gol) penaltıdan gelirken, $\% 13.61$ 'inin (23 gol) serbest vuruş, \%13.02'sinin (22 gol) köşe vuruşu ve \%1.18'inin de (2 gol) taç atış1 sonrasında olmak üzere, maç başına 2,64 gol ortalamas1 ile 2018 FIFA Futbol Dünya kupasinda atılan toplam 169 golün 68'inin \%40.24) duran toplar sonrasında gerçekleştiği belirlenmiştir. Turnuvada kullanılan 29 penaltının 21'inin (\%72.41) golle sonuçlandığ1, köşe vuruşu sonrası atılan gollerin \%18.18'inin sol ayakla, \%81.82'sinin sağ ayakla kullanılan

${ }^{1}$ Assoc. Prof., Mugla Sitki Kocman Univesty, Faculty of Sport Sciences, kgoral@mu.edu.tr 
scored after the set-pieces amongst the all goals scored during the World Cup are to be rather important to effect the game and to reserve a considerable place on the scoreboard. Thus, in order to achieve the success through the tournaments and to complete the competitions as aspired, the set-pieces' effective use and, in defense, the countermeasures to exercise against the set-pieces of opponent teams emerges as a crucial matter. Hereby, it is momentously suggested to accommodate more tactical trainings about set-pieces during the trainings as it might most likely to contribute a lot to team performance.

Keywords: Soccer; World Cup; Penalty; Corner; Free kick; Throw-in; Analysis.

(Extended English summary is at the end of this document) köşe vuruşları sonrasında oluşurken, gollerin \%77.27'si kafa vuruşu ile, \%22.73'ü ise ayakla kaydedilmiştir. Sonuç olarak, dünya kupasında atılan goller içerisinde duran top organizasyonları sonrasinda meydana gelen gollerin oldukça önemli bir yer tuttuğu görülmektedir. Takımların turnuvalarda başarılı olabilmesi ve müsabakalardan istenilen sonuçlarla ayrılabilmeleri açısından, duran topların etkili kullanımı ve kullanılan duran toplara karşı alınacak önlemler üzerine hazırlanmış taktiksel antrenmanlara çalışmalarda daha da fazla yer verilmesinin takım performansına oldukça önemli katkılar sağlayacağ1 söylenebilir.

Anahtar Kelimeler: Futbol; Dünya Kupas1; Penalt1; Köşe Vuruşu; Serbest Vuruş; Taç; Analiz.

\section{Giriş}

Tüm dünyada milyonlarca çocuk, genç ve yetişkinin zevkle oynadığı, milyarlarca insanın seyirci olarak katıldığ1, aniden oluşan süpriz pozisyonlara uyum göstererek doğru kararlar vermeyi gerektiren ve dünyanın en popüler sporu olarak görülen futbolda, bir maçın skorunu değiştirmede ya da kendi lehine çevirmede oldukça önemli etkenlerden birisi de duran toplardır. Bu nedenle duran top organizasyonlarının iyi düzeyde bilinmesi ve çalışlması gerekliliği ortaya çıkmaktadır (Demirci, 1995; İnal, 2004; Kuzucanl, 2008; Gülen, 2013).

Günümüz futbol oyununda temeli bilimsellik üzerine kurulmuş olan analiz yöntemlerinin önemi oldukça artmıştır. Futbol kulüplerinin günümüz teknolojisinin tüm olanaklarından faydalanmalarının yanı sıra saha performanslarının artırılması ve başarıya ulaşılmasında da müsabaka analizi vazgeçilmez bir değerlendirme yöntemi olarak görülmektedir (Dinçer ve ark., 2017). Müsabaka analizi, antrenörlere tarafsız şekilde bazı veriler sunan ve yol gösterici olan bir araçtır (İmamoğlu, Bostanc1, Karaday1 ve İmamoğlu, 2015). Müsabaka analizleri, performans çıtıları yönünden, bireylerin ya da grupların maç sırasındaki aksiyonlarını branşın teknik terimlerine göre düzenleyen, sporcular ve antrenörler ile ilişkili spor davranışlarını tanımlamaktadır (McGarry ve Franks, 2003). Bir müsabakanın analizi yapılarak, tek tek sporcuların ve takımın maç performansları hakkında objektif bilgilere ulaşılabilir. Günümüzün gelişmiş maç analiz sistemleri, oldukça fazla sayıda kare yakalama özelliğine sahip birçok kamera ile görüntülenen maç performanslarının özel bilgisayar programlanı aracılı̆̆ı ile değerlendirilmesi esasına dayanmaktadır (Günay, Şıktar ve Şıktar, 2017). Pozisyona bağlı izleme üzerine kurulu veri teknolojilerinin kullanımıyla gerçek zamanlı kişisel ve anlık oluşabilen geri bildirim firsatlanı, elit spor ortamlanında antrenman ve maçlanın nasıl organize edilebileceğini yönlerdirebilme potansiyeline sahiptir. Bu sebeple, pozisyon verileri, elit sporlardaki geleneksel veri kayıt yaklaşımlarına önemli bir katkı olarak görülebilir (Memmert ve Rein, 2018).

Güncel teknolojik gelişmeler, futbolda analiz alanına etkili bir şekilde destek vermeye çalışan araştırma projelerinin sayısının artmasında da önemli rol oynamışıı (Abreu ve ark., 2012). Futbol, müsabaka analizi alanında, maç anındaki pozisyon verilerine dayanan taktiksel davranışların değerlendirilmesi ve otomatik olarak analiz edilmesi için önemli bir araç haline gelmiştir. İyi planlanmış değerlendirme sistemleri ile çok miktarda verininin az çaba harcayarak ve çok kısa bir 
zamanda elde edilebilmesi, futbolda oyun kurgusu ve uygulamaları için önemli ve yeni değişiklikleri de beraberinde getirebilir (Perl, Grunz ve Memmert, 2013; Fidan ve Yıldız, 2017). Futbol üzerine yapılan bilimsel çalışmalar, 1970'lerde tamamlanan literatür araştırmalar kökenine sahiptir. Bu erken çabalardan beri futbolla ilgili mevcut bilimsel bilgi tabanı önemli ölçüde gelişmiştir. Ortaya konulan bu bilimsel bilgilerin, futbol organizasyonlarını, özellikle de elit takımların günlük çalışmalarını etkilemesi büyük ölçüde kabul görmüştür (Drust ve Green, 2013).

Futbolda, önceden belirlenmiş ölçütler baz alınarak ve objektif veriler elde edilerek yapılan müsabaka analizleri, antrenörlere önemli ölçüde olumlu katkılar sağlayacaktır (Göral ve Saygın, 2012). Futbol üzerine yapılan analizler en fazla gol üzerine gerçekleştirilmektedir. Günümüzde gelişen vuruş teknikleri ile birlikte ise duran toplarla gole ulaşma sayısında da artış olduğu görülmektedir (Çoban, Taşkın, Marangoz ve Akgül, 2016). Futbolda, etkili bir şekilde kullanılan köşe vuruşları ile gol atma arasındaki doğru orantı, takımların başarısında artışa sebep olabilir (Zileli, Söyler ve Genç, 2017). Bu bağlamda, bu çalışmanın amacı, Rusya'da düzenlenen 2018 Dünya kupasındaki müsabakaların analiz edilerek, futbolda duran topların, maçların sonucu üzerindeki etkisinin incelenmesidir.

\section{Yöntem}

Rusya'da düzenlenen 2018 FIFA Dünya kupasında oynanan 64 müsabakada atılan toplam 169 golün duran toplar açısından incelenmesi amacıyla yapılan bu çalışmada, müsabakalarda kullanılan 29 penaltı vuruşu ile kullanılan duran top sonrası golle sonuçlanan 23 serbest vuruş, 22 köşe vuruşu ve 2 taç atışının analizi yapılmışır. Araştırma kapsamında incelenen veriler, FIFA (Fédération Internationale de Football Association) resmi internet sitesinden elde edilmiştir.

Müsabakalarda, penaltı, serbest vuruş, köşe vuruşları ve taç atışları sonrasında gelen gollerin sayıları gibi teknik parametreler e-Analyze Soccer analiz programı kullanılarak, turlara göre penaltılar ve penaltı kullanan oyuncuların ayak tercihleri, turlara göre serbest vuruş sonrası atılan goller ve vuruşları kullanan oyuncuların ayak tercihleri, köşe vuruşu sonrası attlan gollerin vuruş türleri ve vuruşları kullanan oyuncuların ayak tercihleri ise el notasyon sistemiyle analiz edilmiştir.

e-Analyze Soccer analiz programı, video üzerinden analiz yapmaya yarayan bir futbol analiz programıdır. Bu program ile futbolda, bireysel ve takım olarak, oyuncu verimlilik oranları ile istatistikleri gibi özelleştirilmiş raporlar elde edilmektedir. e-Analyze Soccer analiz programinda, müsabaka videoları oynat durdur yöntemi kullanılarak ve bireysel oyuncu istatistikleri için eylemin gerçekleştiği yeri temsili olarak verilen saha üzerinde işaretleme yolu ile analiz yapilır (www.espor.com.tr). El notasyon sisteminde ise, veriler daha önceden hazırlanmış maç izleme kriter cetveli ve analiz formuna manuel olarak el ile yazılır. Bu form, belirlenen değişkenlerin sayısına göre karelere bölünmüş şekildedir (İmamoğlu, Çebi ve Kılcıgil, 2006).

Penaltı: Bir oyuncunun, kendi ceza alanı içerisinde ya da oyunun bir parçası olarak kabul edilen oyun alanının dışında, direkt serbest vuruş gerektiren bir ihlal yapması sonucu verilen atıştır ve bu atıştan doğrudan gol yapılabilir (www.theifab.com/laws).

Köşe vuruşu (Korner): Topun tamamının, son olarak savunma yapan takımın oyuncularından birine dokunarak oyun alanını kale çizgizinden terketmesi sonucu verilen atıştır (www.theifab.com/laws).

Serbest vuruş: Direkt ve endirekt serbest vuruş olarak ikiye ayrilir. Direkt serbest vuruştan doğrudan doğruya gol yapılabilirken, endirekt serbest vuruşta ise gol olabilmesi için vuruşu kullanan oyuncu topa dokunduktan sonra başka bir oyuncu tarafindan daha oynanmalıdır (www.theifab.com/laws).

Taç atış: Topun tamamının yerden ya da havadan taç çizgisini geçtiği durumlarda, topun oyun alanının dışına çıktığı yerden, topa en son dokunan futbolcunun rakibi tarafindan yapılan atıstır (www.theifab.com/laws).

Verilerin analizi: Elde edilen veriler SPSS 18.0 programında kaydedilmiş, frekans ve yüzde değerleri hesaplandıktan sonra yorumlanmıştır. 
Göral, K. (2019). The importance of set-pieces in soccer: Russia 2018 FIFA World Cup analysis. Journal of Human Sciences, 16(3), 885-893. doi:10.14687/jhs.v16i3.5758

\section{Bulgular}

Tablo 1. Duran toplardan atılan gollerin turnuvada atılan toplam gol sayısina oranı

\begin{tabular}{|c|c|c|c|c|c|c|c|c|c|c|}
\hline $\begin{array}{c}\text { Oynanan maç / Turnuvada } \\
\text { atılan toplam gol }\end{array}$ & & nalt1 & & $\begin{array}{l}\text { best } \\
\text { Iruş }\end{array}$ & & $\begin{array}{l}\text { öşe } \\
\text { ruşu }\end{array}$ & & $\begin{array}{l}\Gamma a c ̧ \\
\text { tış1 }\end{array}$ & & plam \\
\hline \multirow{2}{*}{64 / 169} & $\mathrm{f}$ & $\%$ & $\mathrm{f}$ & $\%$ & $\mathrm{f}$ & $\%$ & $\mathrm{f}$ & $\%$ & $\mathrm{f}$ & $\%$ \\
\hline & 21 & 12.43 & 23 & 13.61 & 22 & 13.02 & 2 & 1.18 & 68 & 40.24 \\
\hline
\end{tabular}

2018 Dünya kupasında maç başına 2,64 gol ortalaması ile oynanan 64 maçta toplam 169 gol atılmıştır. Turnuvada atılan gollerin 21'i (\%12.43) penaltıdan, 23'ü (\%13.61) serbest vuruş, 22'si (\%13.02) köşe vuruşu ve 2'si (\%1.18) de taç atışı sonrasında olmak üzere turnuvada attlan toplam 169 golün 68’i (\%40.24) duran toplar sonrasında gerçekleşmiştir.

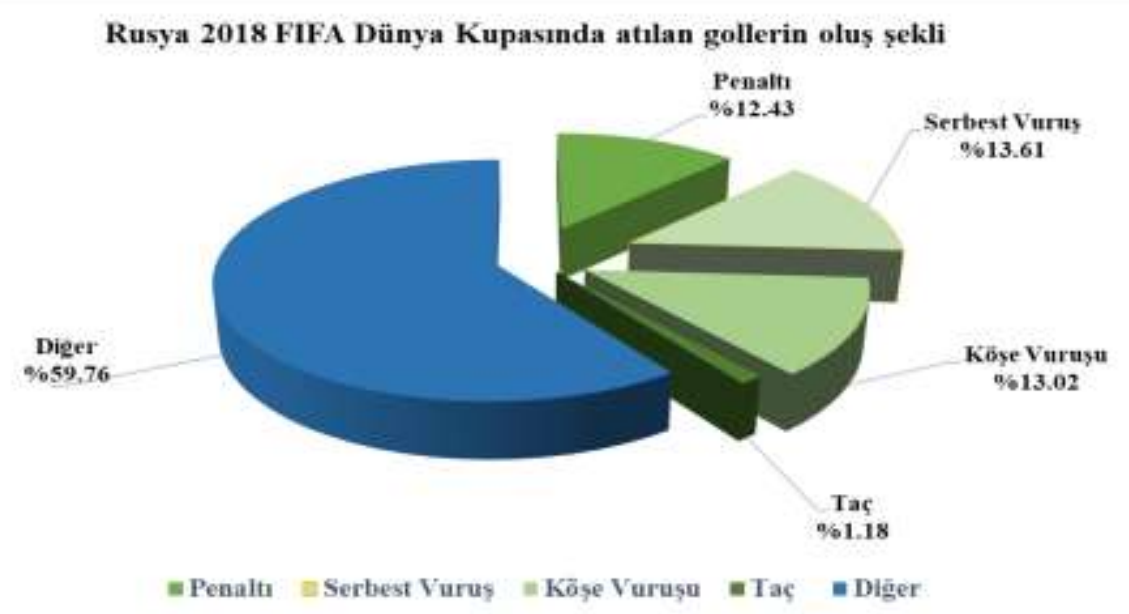

Grafik 1: Rusya 2018 FIFA Dünya Kupasında duran toplar sonrası atılan gollerin oluşumu

Tablo 2. Turnuvada kullanılan penaltılar

\begin{tabular}{cccccc}
\hline Kullanılan penaltı / Oynanan maç & \multicolumn{2}{c}{ Golle sonuçlanan Penaltı } & \multicolumn{2}{c}{ Kaçan penaltı } \\
\hline \multirow{2}{*}{$29 / 64$} & $\mathrm{f}$ & $\%$ & $\mathrm{f}$ & $\%$ \\
\cline { 2 - 5 } & 21 & 72.41 & 8 & 27.59 \\
\hline
\end{tabular}

Turnuvada oynanan 64 müsabakada toplam 29 penaltı kararı verilirken, kullanılan 29 penaltıdan 21’i (\%72.41) golle sonuçlanmıştır. 8 penaltı ise gole çevrilememiştir (\%27.59).

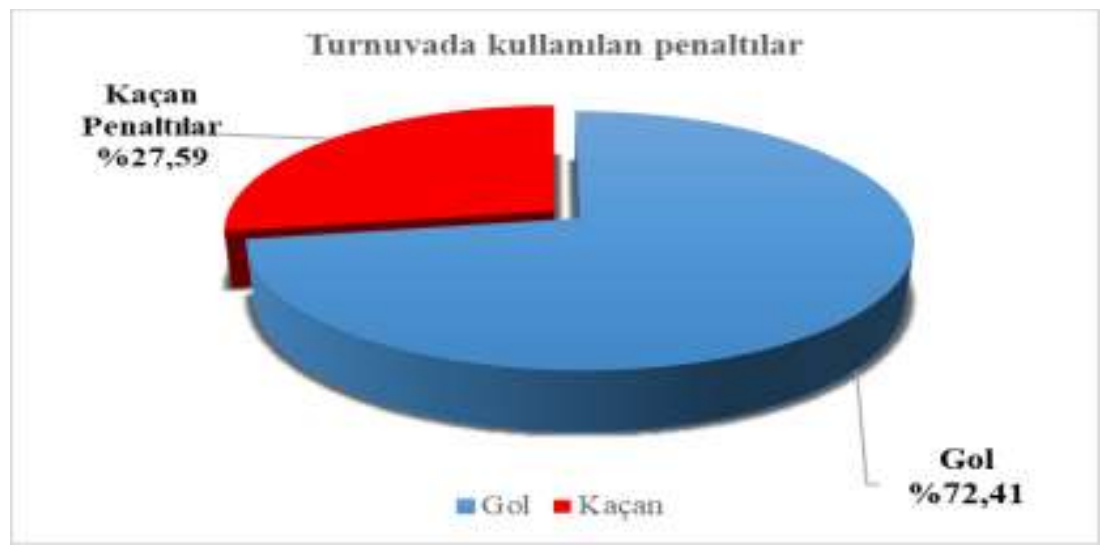

Grafik 2: Rusya 2018 FIFA Dünya Kupasında kullanılan penaltılar 
Göral, K. (2019). The importance of set-pieces in soccer: Russia 2018 FIFA World Cup analysis. Journal of Human Sciences, 16(3), 885-893. doi:10.14687/jhs.v16i3.5758

Tablo 3. Turlara göre penaltılar ve penaltı kullanan oyuncuların ayak tercihleri

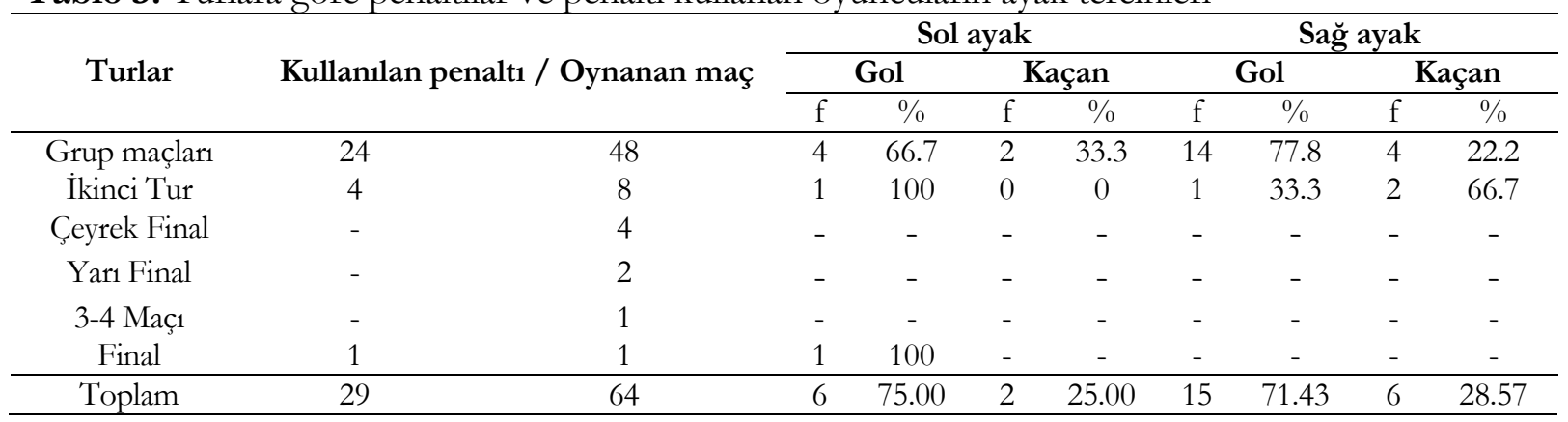

Turnuvada oynanan 48 grup maçında maç başına 0,50 penaltı ile toplam 24 penaltı vuruşu kullanılırken, eleme turunun başlangıcı olan ikinci tur maçlanında da aynı şekilde maç başına 0,50 penaltı ile toplam 4 penaltı kullanılmıştır. Çeyrek final, yarı final ve 3.lük-4.lük maçında penaltı kararı verilmezken, final maçındaki 1 penaltı vuruşu ile turnuva genelinde kullanılan penaltı sayısı maç başına 0,45 penaltı ile 29 olmuştur.

Tablo 4: Turlara göre serbest vuruş sonrası atılan goller ve vuruşları kullanan oyuncuların ayak tercihleri

\begin{tabular}{ccccccc}
\hline Turlar & \multicolumn{2}{c}{$\begin{array}{c}\text { Serbest vuruş sonrası } \\
\text { atılan gol / Oynanan maç }\end{array}$} & \multicolumn{2}{c}{$\begin{array}{c}\text { Sol ayak } \\
\text { Gol }\end{array}$} & \multicolumn{2}{c}{$\begin{array}{c}\text { Sağ ayak } \\
\text { Gol }\end{array}$} \\
\hline & & & $\mathrm{f}$ & $\%$ & $\mathrm{f}$ & $\%$ \\
Grup maçlar1 & 17 & 48 & 6 & 35.29 & 11 & 64.71 \\
İkinci Tur & 1 & 8 & 1 & 100 & - & - \\
Çeyrek Final & 2 & 4 & 1 & 50.00 & 1 & 50.00 \\
Yar1 Final & 1 & 2 & - & - & 1 & 100 \\
3-4 Maç1 & - & 1 & - & - & - & - \\
Final & 2 & 1 & 1 & 50.00 & 1 & 50.00 \\
\hline Toplam & 23 & 64 & 9 & 39.13 & 14 & 60.87 \\
\hline
\end{tabular}

Turnuvada oynanan 64 müsabakada, maç başına 0,35 gol ortalaması ile atılan 169 golün 23’ü (\%13.61) serbest vuruş sonrasında gerçekleşmiştir. Grup maçlarında atılan gollerin \%35.29’u sol ayakla, \%64.71'i ise sağ ayakla kullanilan servest vuruşlar sonrası atılırken, toplamda atılan 23 golün 9’u (\%39.13) sol ayakla, 14’ü (\%60.87) sağ ayakla oyuna sokulan toplar sonrası meydana gelmiştir.

Tablo 5: Köşe vuruşu sonrası atılan goller, gol vuruşu türleri ve vuruşları kullanan oyuncuların ayak tercihleri

\begin{tabular}{cccccccccc}
\hline \multirow{2}{*}{$\begin{array}{c}\text { Köşe vuruşu sonrası } \\
\text { atılan gol / Oynanan maç }\end{array}$} & \multicolumn{2}{c}{ Sol ayak } & \multicolumn{2}{c}{ Sağ ayak } & \multicolumn{3}{c}{ Gol vuruşu } \\
\cline { 2 - 10 } & \multicolumn{2}{c}{ Gol } & \multicolumn{2}{c}{ Gol } & \multicolumn{2}{c}{ Kafa } & \multicolumn{2}{c}{ Ayak } \\
\cline { 2 - 10 } & $\mathrm{f}$ & $\%$ & $\mathrm{f}$ & $\%$ & $\mathrm{f}$ & $\%$ & $\mathrm{f}$ & $\%$ \\
\hline 22 & 64 & 18.18 & 18 & 81.82 & 17 & 77.27 & 5 & 22.73 \\
\hline
\end{tabular}

Turnuvada oynanan 64 müsabakada, maç başına 0,34 gol ortalaması 169 golün 22'si (\%13.02) kösse vuruşları sonrasında gerçekleşmiştir. Müsabakalarda attlan gollerin \%18.18’i sol ayakla, \%81.82'si sağ ayakla kullanılan köşe vuruşları sonrasında oluşurken, gollerin \%77.27'si kafa vuruşu ile, \%22.73’ü ise ayakla kaydedilmiştir.

\section{Tartışma ve Sonuç}

Futbolda penalt1, serbest vuruş, köşe vuruşu ve taç atışı sonrası oluşan gollerin incelenmesi amacıyla Rusya'da düzenlenen 2018 Dünya kupasındaki müsabakaların incelendiği bu çalışmada, turnuvada oynanan 64 müsabakada gole dönüşen 21 ve kaçan 8 penaltı olmak üzere kullanılan 29 penaltı (maç başına 0,45), kullanılan duran top sonrasında gol olarak sonuçlanan 23 serbest vuruş (maç başına 0,36), 22 köşe vuruşu (maç başına 0,34) ve 2 taç atışının (maç başına 0,03) analizi yapılmıstur. Turnuvada oynanan müsabakalarda, atllan gollerin \%12.43’ünün penaltıdan, \%13.61’inin 
serbest vuruş, \%13.02'sinin köşe vuruşu ve \%1.18’inin de taç atışı sonrasında olmak üzere 2018 FIFA Futbol Dünya kupasinda atılan tüm gollerin \%40.24’ünün duran toplar sonrasında olduğu tespit edilmiştir.

Cerrah ve Gürol (2011) Türkiye Futbol Süper Liginde 2001-2009 yılları arasında oynanan maçlarda atılan gollerin analizini yaptıkları çalışmada, tamamlanan 8 sezonda atılan gollerin \%30'unun (2.028 gol) duran toplar sonrası meydana geldiğini belirtmişlerdir. Çoban, Taşkın, Marangoz ve Akgül'ün (2016) şampiyonlar ligi final maçlarında kullanılan duran topları inceledikleri çalışmada, kazanan takımlar rakip sahada kazandıkları 61 duran topun sadece 2 tanesini gole dönüştürebilirken, kaybeden takımlar rakip sahada kazandıkları 43 duran topun hiç birinde gole ulaşamamışlardır. Sönmeyenmakas (2008) UEFA Avrupa Şampiyonlar Liginde atılan golleri analiz ettiği çalışmasında, 2004/2005, 2005/2006 ve 2006/2007 sezonlarında sirasıyla atılan gollerin \%19.7 (65 gol), \%27.8 (79 gol) ve \%23.9’unu (74 gol) oluşturduğunu bulmuştur.

Leite (2013) Euro 2012'de atılan gollerin analizini yaptığı çalışmada, turnuvada atılan 76 golün 22'sinin (\%28,95); Njororai (2013a) ise Güney Afrikada düzenlenen 2010 Dünya kupasinda atılan 145 golün 35'inin (\%24.14) duran toplar sonrasında geldiğini belirtmektedir. Armatas ve Yiannakos (2010) 2006 Dünya Kupasında atılan gollerin analizini ve değerlendirilmesini yaptıkları çalışmada, turnuvada atılan gollerin \%32,6'sının penaltı, köşe vuruşu, serbest vuruş ve taç atışı gibi set oyunları sonrasında geldiğini belirtmişlerdir.

Ziyagil ve Çebi (2000) Fransa 1998 Dünya Kupasını analiz ettikleri çalışmada, turnuvada atılan gollerin \%10.53'ünü penaltıdan ve serbest vuruştan atılan gollerin oluşturduğunu; Ali, Katis, Patsika ve Kellis (2015) Yunan futbol liginin sekiz sezonunu analiz ettikleri çalışmada, atılan gollerin \%8,1'inin (367 gol) penaltıdan, \%4,2'sinin (187 gol) de frikik sonrasında gerçekleştiğini tespit etmişlerdir. Daha önce yapılan bu çalışmalara göre, 2018 FIFA Futbol Dünya kupasında penaltıdan atılan gol oranının daha yüksek çıkmasında, FIFA Dünya Kupasında da uygulanan VAR (Video Yardımcı Hakem) uygulamasının oldukça önemli bir etkisinin olduğu söylenebilir.

Njororai (2013b) 2002 Dünya kupasında atılan gollerin \%28,6'sinın, 1994 Dünya kupasında atılan gollerin ise \%33,3'ünün duran toplar sonunda geldiğini belirtmektedir. Gülen (2014) Brezilyadaki 2014 Dünya Kupasında oynanan 64 müsabakada atılan gollerin \%29'unu oluşturan toplam 50 golün duran toplar sonrasında gerçekleştiğini, bu gollerin 12'sinin penaltılardan, 12'sinin serbest vuruşlardan ve 26'sının da kösse vuruşlanı sonrası geldiğini belirtmiştir. Cerrah, Özer ve Bayram (2016) duran toplardan atılan gollerin niceliksel analizini yaptıkları çalısmada, Türkiye Süper Liginde 2006-2011 yılları arasındaki 5 sezonda duran top sonrası atılan gollerin sayısının tüm gollere oranını \%28,22 olarak bulmuşlardır. Yiannakos ve Armatas (2006) Portekizde düzenlenen 2004 Avrupa Şampiyonasında (Euro 2004) atılan gollerin \%35,6'sının duran toplar sonunda geldiğini tespit etmişlerdir. Elde edilen bu sonuçlar, çalışmada ortaya çıkan bulgularla paralellik göstermektedir.

Duran toplardan etkili bir şekilde yararlanabilmek için, bireysel çalışmaların haftalık antrenman programının temel bir parçası haline gelmesi gerekmektedir. Ayrıca, duran top antrenmanları, yalnızca gol atma ve gol atmaya yönelik firsatlar, pozisyonlar açığa çıkarma üzerine odaklanmamalı, savunmaya yönelik bir bakış açısı ile de ele alınmalıdır (Armatas \& Yiannakos, 2010).

Turnuvada, köşe vuruşu sonrasında gelen gollerin \%77.27'sinin kafa vuruşu ile atılmış olması, duran toplarda hava hakimiyeti yüksek olan oyuncuların takıma olan katkılarının önemli bir göstergesi olarak değerlendirilebilir. Sonuç olarak, ortaya çıkan bulgulardan yola çıkarak, duran top organizasyonları sonrasinda meydana gelen gollerin, futbolun en büyük turnuvası olarak bilinen dünya kupasında atılan gollerde oldukça önemli bir yer kapladığı, futbolun hangi kademesinde olursa olsun, takımların başarıya ulaşabilmeleri açısından, duran topların kullanımı ve alınacak önlemler üzerine, iyi bir planlama yapılarak hazırlanmış taktik çalışmalara futbolcuların antrenmanlarında daha da fazla yer ayrılmasının takımın ortaya koyacağı performansa önemli ölçüde olumlu katkılar sağlayacağı söylenebilir. 


\section{Kaynaklar}

Abreu, P. H., Moura, J., Silva, D. C., Reis, L. P., \& Garganta, J. (2012). Performance analysis in soccer: A Cartesian coordinates based approach using RoboCup data. Soft Computing, 16(1): 47-61.

Ali, M. F. M., Katis, A., Patsika, G., \& Kellis, E. (2015). Goal scoring charateristics in soccer: Are they technique and time dependent? Asia Pacific Journal of Advanced Business and Social Studies. 1(1): 186-194.

Armatas, V., \& Yiannakos, A. (2010). Analysis and evaluation of goals scored in 2006 World Cup. Journal of Sport and Health Research. 2(2): 119-128.

Cerrah, A.O., \& Gürol, B. (2011). Türkiye Futbol Süper Ligi’nde 2001-2009 Yıllarn Arasinda Atulan Gollerin Analizi. Türkiye Klinikleri Spor Bilimleri Dergisi, 3(2): 79-85.

Cerrah, A.O., Özer, B., \& Bayram, İ. (2016). Duran Toplardan Atılan Gollerin Niceliksel Analizi: Türkiye Süper Lig Uygulaması. Türkiye Klinikleri Spor Bilimleri Dergisi, 8(2): 37-45.

Çoban, O., Taşkın, A.K., Marangoz, İ., \& Akgül, F. (2016). Son Dört Yllın Şampiyonlar Ligi Final Maçlarının Duran Top ve Gol Analizi. The Journal of Academic Social Science, 4(36): 719-726.

Demirci, N. (1995). A'dan Z'ye Spor. Ankara: Neyir Yayıncilik ve Matbaacilik, s.202.

Dinçer, Ö., Arı, E., Sözen, H., Erdoğan, E., \& Cevahircioğlu, B. (2017). Profesyonel Futbol Takımlarının Alt Yapılarında Oynayan Genç Futbolcuların Profesyonel Futbol Ligleri için Performans Açısından Yeterlilik Düzeylerinin İncelenmesi. Spor ve Performans Arașttrmalar Dergisi, 8(1): 35-46.

Drust, B. \& Green, M. (2013). Science and football: Evaluating the influence of science on performance. Journal of Sports Sciences, 31(13): 1377-1382.

Fidan, U., \& Y1ldı, M. (2017). Reliability and validity of the new shooting accuracy measurement (SAM) system software. Journal of Sports Science, 5, 172-177.

Göral, K., \& Saygın, Ö. (2012). Birinci ligde yer alan bir futbol takımının sezon performansının incelenmesi. Uluslararasi Insan Bilimleri Dergisi, 9(2): 1017-1031.

Gülen, N.M. (2013). Duran Toplar. Futbol Gelişim, 6: 12.

Gülen, N.M. (2014). Serbest Vuruşlar (Hücum). Futbol Gelişim, 9: 16-17.

Günay, M., Şıktar, E., \& Şıktar, E. (2017). Antrenman Bilimi. Batman Belediyespor Kültür Eğitim ve Spor Yayınları, Ankara: Özgür Web Ofset Matbaacilı, s.797.

İmamoğlu, O., Çebi, M., \& Kılcıgil, E. (2006). 2006 FIFA Dünya Futbol Kupasındaki Gollerin Teknik ve Taktik Kriterlere Göre Analizi. Spormetre Beden Eğitimi ve Spor Bilimleri Dergisi, 5(4):157-165.

İnal, A.N. (2004). Futbolda Eğitim Öğretim. Ankara: Nobel Yayın Dağıtım, s.15.

Leite, W. S. (2013). Euro 2012: Analysis and Evaluation of Goals Scored. International Journal of Sports Science, 3(4): 102-106.

Kuzucanlı, B. (2008). Dünyada 100 Ünlü Futbolcu. İstanbul: Morpa Kültür Yayınları, s.4.

McGarry, T., \& Franks, I.M. (2003). The science of match analysis. In T.Reilly and A.M.Williams (Eds.), Science and Football (2nd Edition). London: Routledge.

Memmert, D., \& Rein, R. (2018). Match Analysis, Big Data and Tactics: Current Trends in Elite Soccer. Deutsche Zeitschrift Für Sportmedizin, 69(3): 65-72.

Njororai, W.W.S. (2013a). Analysis of goals scored in the 2010 world cup soccer tournament held in South Africa. Journal of Physical Education and Sport, 13(1): 6-13.

Njororai, W.W.S. (2013b), Downward Trend of Goal Scoring in World Cup Soccer Tournaments (1930 to 2010). Journal of Coaching Education, 6(1): 111-125

Perl, J., Grunz, A., \& Memmert, D. (2013). Tactics Analysis in Soccer - An Advanced Approach. International Journal of Computer Science in Sport, 12(2013): 33-44.

Sönmeyenmakas, A. (2008). UEFA Şampiyonlar Ligi’nde Atılan Gollerin Analizi. Trakya Üniversitesi Sağlık Bilimleri Enstitüsü Beden Eğitimi ve Spor Anabilim Dalı Yüksek Lisans Tezi, Edirne. 
Göral, K. (2019). The importance of set-pieces in soccer: Russia 2018 FIFA World Cup analysis. Journal of Human Sciences, 16(3), 885-893. doi:10.14687/jhs.v16i3.5758

Yiannakos, A., \& Armatas, V. (2006). Evaluation of the goal scoring patterns in European Championship in Portugal 2004. International Journal of Performance Analysis in Sport, 6(1):178188.

Zileli, R., Söyler, M., \& Genç, A. (2017). 2016-2017 Turkcell Süper Liginde Oynanan Maçlarda Kullanılan Köşe Vuruşlarının Maç Analizi. Inönü Üniversitesi Beden Eğitimi ve Spor Bilimleri Dergisi, 4(3):48-58.

Ziyagil, M.A., \& Çebi, M. (2000). 1998 Fransa Futbol Dünya Kupasındaki Gollerin Lateralite, Teknik ve Taktik Kriterlere Göre Analizi. Futbol Bilim ve Teknoloji Dergisi, 7(4):18-23.

http://www.theifab.com/document/laws-of-the-game. Erişim tarihi: 03.10.2018

http://www.espor.com.tr/kitapciklar/eanalyze soccer tr.pdf. Erişim tarihi: 20.11.2018

https://www.fifa.com/worldcup/videos/match-highlights/. Erişim tarihi: 14.08.2018

\section{Extended English Summary}

Footbal, is a game that requires its players to make certain and appropriate decisions against the unexpected incidents happens during playtime; also, is a sport which millions of children, young and adult people enjoy playing and billions of humans attending as spectators. One of the significant ways to change the score on the board in favour of team is the set-piece. Therefore, to win the game, the set-piece organizations must be well known and well practiced (Demirci, 1995; İnal, 2004; Kuzucanl1, 2008; Gülen, 2013). In today's football, the importance of analysis methods based on scientifically significant calculations has rather icreased. Alongside the utilization of all the technological resources for football clubs to achive success, competition analysis is regarded as an indispensable assessment and evaluation procedure (Dinçer at al., 2017). In football, competition analysis, which is prepared as pre-configured measures and objective datas considered, helps trainers and contribute to team-play towards winning the game. (Göral and Saygin, 2012). Most football analysis executed as scores taken into consideration. Lately, as shooting styles and techniques improves, rates of scoring goals from set-pieces increases as well (Çoban, Taşkın, Marangoz and Akgül, 2016). Especially, if counted on the relation and ratio of successfully executed corner-kick positions and achieving scores, this may help teams reaching and increasing the victory (Zileli, Söyler and Genç, 2017). Penalties, free-kicks (direct-indirect), throw-ins, corner kicks and goal kicks are known to be as set-pieces. In this study; examination of set-pieces' effects on scoring goals were targeted and, in this context, the competitions of Russia 2018 FIFA World Cup were analyzed.

As a part of this study, 64 competitions of Russia 2018 FIFA World Cup were analyzed depending on penalties and set-pieces (free-kicks, corner kicks and throw-ins etc.) ending with goals. "E-Analyze Soccer" computer program were used to analyze the set-piece organizations in the competitions at the tournament. The findings were registered and interpreted via SPSS (18.0) program upon calculations of frequencies and percentages. $21(\% 12.43)$ of the all goals scored in this tournament were from penalties, 23 (\%13.61) from free-kicks, 22 (\%13.02) from corner kicks and $2(\% 1.18)$ from throw-ins. Throughout the Russia 2018 FIFA World Cup, 169 goals were scored in total with an average of 2.64 goals per game and, $68(\% 40.24)$ of all the goals were recorded as score following the set-pieces. In the tournament, $21(\% 72.41)$ penalties out of 29 resulted with goals. A major part of the goals scored from corner kicks were completed via headings (\%77.27) and rest with kicks (\%22.73). A great amount of completing goal scoring kicks following corner kicks were recorded with right foot (\%81.82) and the rest with left foot (\%18.18). Leite (2013), analysed goals scored in Euro 2012, and found that $22(\% 28,95)$ of 76 goals actualized from set-pieces; also Njororai (2013a), found that in 2010 World Cup, 35 (\%24.14) of 145 goals scored following set-pieces. Armatas and Yiannikos (2010) in their research of 2006 World Cup, found \%32.6 of all goals scored following set-pieces such as penalties, corner kicks, free kicks, 
throw-ins etc. Ziyagil and Çebi (2000) found that in France 1998 World Cup, \%10.53 of all goals scored from penalties and free-kicks; Ali, Katis, Patsika and Kellis (2015) researched the Greek Football League for 8 seasons and found that \%8.1 (367) of all goals scored after penalties, and $\% 4.2$ (187) of all goals occured following the free-kicks. In their research of Champions League, Marangoz and Akgül (2016) found that, throughout the tournament, winning teams turned only 2 set-piece positions of all 61 into goals while in their opponents' field; and losing teams found no goals in all of 43 set-piece positions while in their opponents' field. Gülen (2014) in the research on goals scored during 2014 World Cup in Brasil, found that \%29 (50) of all goals achieved following the set-pieces throughout the 64 games. Particularly, 12 of these goals scored after penalties, 12 after free-kicks and 26 following the corner kicks. As a result, the goals scored after the set-pieces amongst the all goals scored during the World Cup are to be rather important to affect the game and to reserve a considerable place on the scoreboard. Thus, to achieve the success through the tournaments and to complete the competitions as aspired, the set-pieces' effective use and, in defense, the countermeasures to exercise against the set-pieces of opponent teams emerges as a crucial matter. Hereby, it is momentously suggested to accommodate more tactical trainings about set-pieces during the trainings as it might most likely to contribute a lot to team performance. 